\title{
UN NUEVO DEPOSITO DE ARMAS DEL BRONCE FINAL EN EL RIO GUADALQUIVIR
}

\author{
POR
}

MANUEL M. RUIZ DELGADO (*)

RESUMEN Presentamos un nuevo depósito de armas pertenecientes al Bronce Final hallado en el Río Guadalquivir en los trabajos de dragado del mismo a principios de los años ochenta.

Consta de una espada de lengua de carpa y de una punta y un regatón de lanza, todas de bronce. Según los datos documentados hasta ahora para los demás depósitos andaluces de estas características, su vinculación con zonas acuáticas parece confirmarse casi en la totalidad de los casos, constituyendo un dato importante más, favorable a un nuevo planteamiento de las costumbres, probablemente funerarias, de este momento cultural de Andalucia.

ABSTRACT This paper describes a new Late Bronze Age weapon hoard from the river Guadalquivir (prov. Seville, Spain). The hoard consists of a carp's tongue sword, a ferrule, and a socketed spearhead.

The absence of good archaeological contexts for this kind of weapon hoard has made dating them difficult, but recently a carp's tongue sword has been found in a stratigraphic context with a date of $950-850$ B. C.

These finds suggest perhaps that there is some relation between these funerary weapon hoards and water (rivers, lakes, the sea, etc.).

Palabras clave: Depósito de armas del Bronce Final. Río Guadalquivir. Espada lengua de carpa. Punta y regatón de lanza. Costumbres funerarias.

Late Bronze Age weapon hoard. Guadalquivir river. Carp's tongue sword, ferrula, socketed spearhead. Funerary weapon hoard.

El número creciente de objetos metálicos de variada índole aparecidos en el Sur de la Península Ibérica, atribuidos a las diversas fases del Bronce Final, tanto procedentes de hallazgos aislados como de depósitos, empiezan a avalar la necesidad, ya intuida por algunos investigadores (Carrasco et alii, 1987), de un nuevo planteamiento sobre la importancia económica y el desarrollo de la metalurgia en nuestra región en este momento.

Desde el conocido conjunto de la Ría de Huelva, en el primer tercio del presente siglo hasta la actualidad, es incesante el número de nuevos hallazgos que continuamente vienen documentándose.

En su inmensa mayoria se trata, desgraciadamente, de objetos que llegan hasta nosotros sin

(") Dep. de Prehistoria y Arqueología de la Facultad de Geografía e Historia. Sevilla. 
contexto arqueológico definido o con contextos poco fiables, o conocidos, y, frecuentemente, sin una ubicación geográfica clara, al proceder muchos de ellos de colecciones privadas.

En los últimos tiempos y en el campo concreto de las espadas, se ha podido documentar un interesantísimo ejemplar de lengua de carpa con cronología y contexto claros aparecido en estratigrafía en el yacimiento granadino del Cerro de la Miel (Moraleda de Zafayona), recientemente publicado en un interesante y documentado estudio (Carrasco et alii, 1987). Constituye hasta ahora uno de los escasísimos ejemplares, si no el único, hallado en estas circunstancias, por lo que hemos de referirnos a él a la hora de estudiar cualquier ejemplar aparecido en nuestra región.

Dentro de este contexto inscribimos este nuevo depósito aparecido en el Guadalquivir, recogiendo en este estudio preliminar los primeros datos sobre las distintas piezas aparecidas hasta ahora, cuyo estudio en profundidad abordaremos proximamente.

\section{EL HALLAZGO}

Por noticias orales sabemos que las tres piezas conocidas hasta ahora de este depósito aparecieron en los trabajos de dragado del fondo del río, a principios de los años ochenta, en una zona equidistante de las actuales poblaciones de la Rinconada y Alcalá del Río en la provincia de Sevilla.

Tenemos noticias también de otros objetos, hoy en paradero desconocido, pertenecientes al mismo depósito, que debió contar, probablemente, con un número, al parecer elevado, de piezas, entre espadas de lengua de carpa, puntas y regatones de lanza, fíbulas, etc.

Muy posiblemente las espadas que publica M. L. Ruiz-Gálvez (1984: 669 y ss) procedentes de Alcalá del Río (Sevilla) provienen de este mismo depósito, ya que tienen un mismo origen fluvial. Todos los objetos a los que se hace alusión en esta breve reseña fueron localizados en un mismo lugar, situado cerca de la orilla derecha del río.

\section{INVENTARIO DE OBJETOS}

Los objetos que hemos podido documentar del conjunto de piezas de bronce aparecidas hasta el momento consisten en una espada de «lengua de carpa», una punta y un regatón de lanza.

1. La espada de «lengua de carpa" o "gota de sebo" se halló en excepcional estado de conservación. Sólo está fragmentada muy levemente en uno de los apéndices o "gavilanes" que conforman el extremo de «lengüeta" de la empuñadura. Está realizada en una sola pieza de fundición. La empuñadura es de lengüeta plana y sección rectangular decreciente hacia la base. Presenta en la parte central y en sentido longitudinal tres horadaciones de tendencia cuadrangular y rectangular. La primera y más cercana a la base es irregular con tendencia rectangular, la central es cuadrangular y la más cercana a la guarda es también de tendencia cuadrangular y de menor tamaño que la anterior. La lengüeta tiene, como ya es clásico en este tipo de espadas, una terminación bífida, formada por un tope con dos apéndices laterales o kgavilanes" perpendiculares a la empuñadura. En la parte superior aparece ésta afilada.

En su parte más próxima a la hoja, la empuñadura se ensancha fuertemente, adquiriendo una forma triangular invertida que da lugar a las denominadas *aletas" de la empuñadura o guarda. Estas aletas contienen, de forma simétricamente dispuestas, cuatro horadaciones (dos de cada lado) 

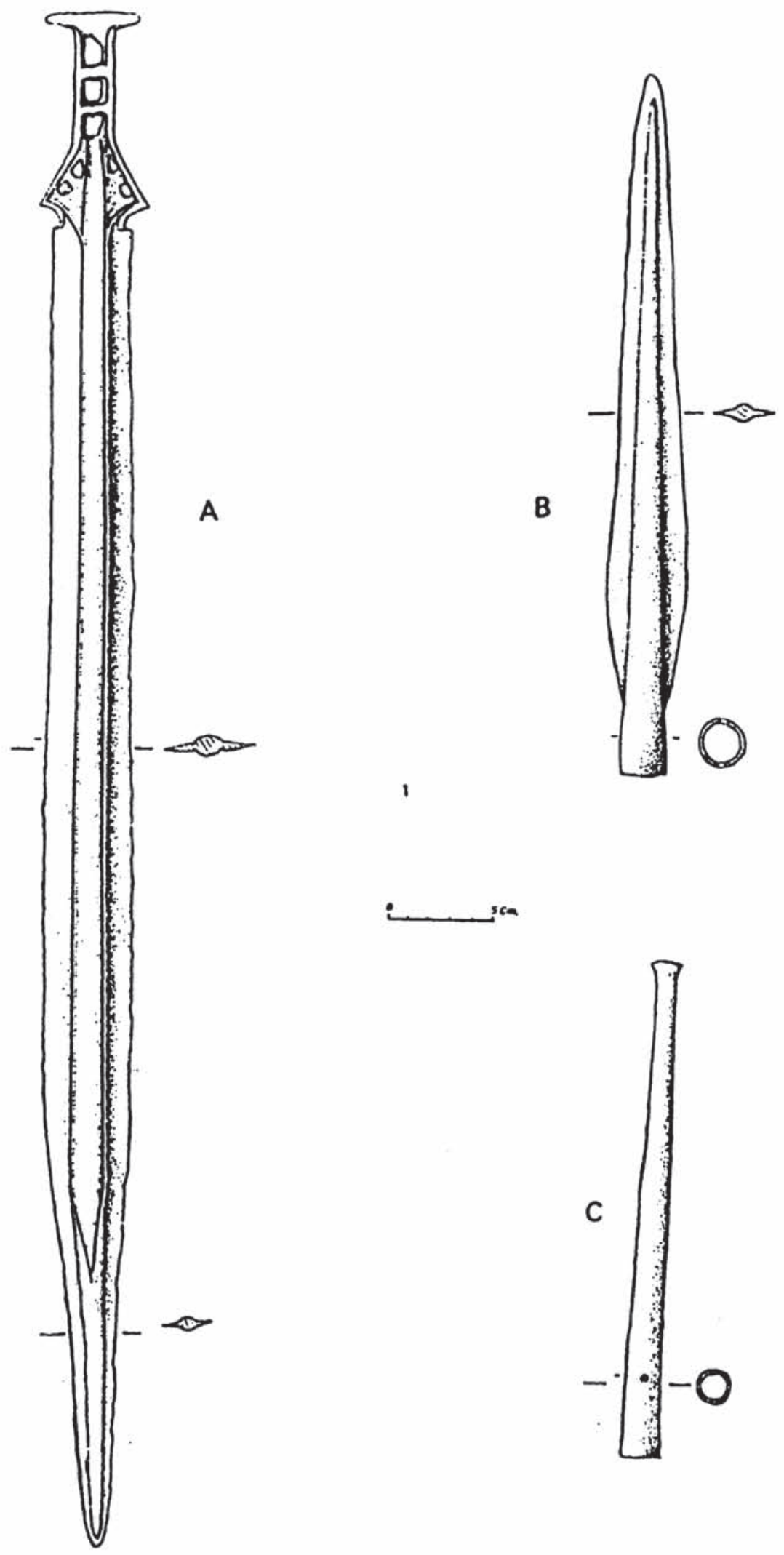

1
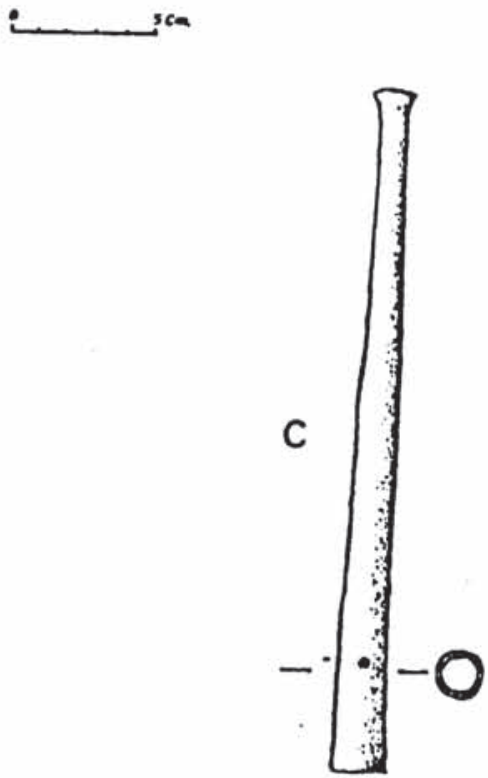

Fig. 1.-Piezas del depósito del Guadalquivir. 
de apariencia ligeramente cuadrangular las más próximas a la hoja, y de apariencia triangular las más próximas a la lengüeta de la empuñadura. Los remaches que posiblemente poseía se han perdido.

Debajo de las aletas se sitúan dos escotaduras espesas que separan la guarda de la hoja propiamente dicha de la espada. Estas escotaduras o *ricassos" describen un cuarto círculo desde los vertices de las aletas hasta el comienzo de la hoja, que es recto y perpendicular al eje longitudinal de la espada.

La hoja no tiene los lados geométricamente rectos, sino que tiene una forma ligeramente pistiliforme, siendo algunos milimetros más ancha justamente donde comienza el tercer tercio de la hoja, si contamos a partir de la zona de la empuñadura. Esta característica parece ser general en este tipo de espadas, ya que se observa en la casi totalidad de los ejemplares documentados hasta ahora. En el punto antes indicado comienza justamente el estrechamiento de la hoja, para acabar formando la punta característica que le da nombre al tipo.

Un fuerte nervio central recorre longitudinalmente la espada desde la base de la última horadación de la lengüeta de la empuñadura, dividiendo en dos las aletas de la guarda, hasta el extremo de la hoja. Todo el nervio está delimitado por dos finas acanaladuras laterales que, partiendo de la parte interior de los vértices de las aletas llegan al inicio del tercer tercio de la hoja, desde donde acaban cerrándose en un ángulo puntiagudo abandonando la delimitación del nervio central que continúa hasta el extremo de la hoja.

Dimensiones: Longitud máxima, $765 \mathrm{~mm}$. Longitud de la empuñadura, $110 \mathrm{~mm}$. Longitud de la hoja $663 \mathrm{~mm}$. Anchura máxima de la lengüeta, $43 \mathrm{~mm}$. Anchura máxima de la hoja, $41 \mathrm{~mm}$. Anchura máxima del nervio central, $15 \mathrm{~mm}$. Anchura máxima de las escotaduras, $10 \mathrm{~mm}$. Sección media de la hoja, $10 \mathrm{~mm}$. (Fig. 1A).

2. Punta de lanza con enmangue tubular corto y gruesa nervadura central. El enmangue conserva dos pequeñas horadaciones perpendiculares para sujetar con clavos el asta de madera que iría engarzada en aquella. La calidad del bronce es idéntica al de la espada precedente. Su estado de conservación es excelente.

Sus dimensiones, como veremos seguidamente, le dan a la punta una esbeltez poco común en piezas de este tipo documentadas hasta ahora en nuestra zona.

Dimensiones: Longitud máxima, $345 \mathrm{~mm}$. Anchura máxima, $39 \mathrm{~mm}$. Anchura de la base, $31 \mathrm{~mm}$. Anchura máxima de aletas, $10 \mathrm{~mm}$. Las horadaciones se sitúan a $13 \mathrm{~mm}$. de la base del enmangue. (Fig. 1B).

3. Regatón de lanza de forma tubular en buen estado de conservación. Su diámetro va disminuyendo conforme se acerca a la base de la pieza, que presenta un ensanchamiento bulboso. $\mathrm{Al}$ igual que en el caso de la punta de lanza, esta pieza presenta también dos pequeñas horadaciones perpendiculares a unos $4 \mathrm{~cm}$. de la parte superior de la misma con el mismo fin que aquélla. No lleva decoración.

Dimensiones: Longitud máxima, $245 \mathrm{~mm}$. Anchura máxima, $19 \mathrm{~mm}$. Anchura mínima, $11 \mathrm{~mm}$. Anchura de la base, $16 \mathrm{~mm}$. (Fig. 1C).

\section{VALORACION GENERAL}

La presencia de este depósito en el río Guadalquivir resulta enormemente interesante, dada su situación geográfica y el carácter y composición del mismo. Muy posiblemente estas piezas, como ya apuntamos anterformente, no constituyen sino una reducida parte de lo que sería el primitivo 


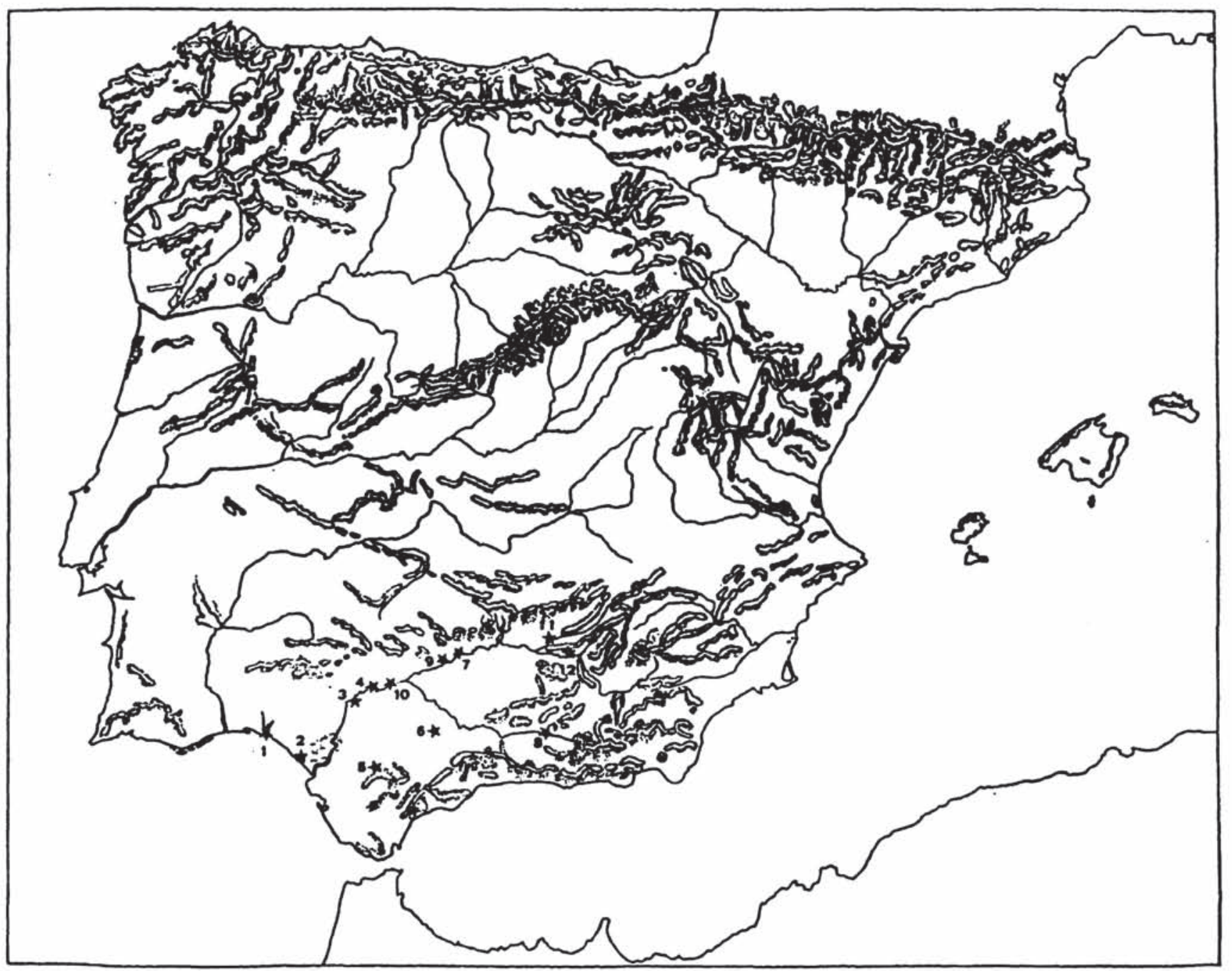

Fig. 2.- Hallazgos andaluces de espadas de lenguas de carpa.

1. Ria de Huelva- 2. Coto de Doñana (Huelva)- 3. La Cartuja (Sevilla).- 4. Entre La Rinconada y Alcala del Río (Sevilla). - 5. Bornos (Cádiz). 6. Los Castellares (Herrera, Sevilla).- 7. Entre Marmolejo y Villa del Río (Córdoba). \& Cerro de la Mora (Moraleda de Zafayona, Granada). 9. Palma del Río (Córdoba)- 10. Alcalá del Ría. (Sevilla). 11. Guadalimar (Jaén). - 12. Baeza (Granada).

depósito. Si sumamos a las tres piezas documentadas últimamente las dos espadas publicadas por M. L. Ruiz-Gálvez (1984: 669 y ss) como procedentes de Alcalá del Río (Sevilla), una de lengua de carpa y otra del tipo de Sa Idda, tenemos ya cinco piezas (tres espadas, una punta y un regatón de lanza) todas de bronce. Esperemos que en el futuro podamos documentar alguna pieza más, hoy en manos de colecciones privadas.

La presencia de este depósito en el fondo del río viene a confirmar, una vez más, la estrecha vinculación que este tipo de objetos del Bronce Final meridional entre otros parece tener con zonas acuáticas tanto fluviales como marítimas.

La gran mayoría de estas piezas, sobre todo armas, pertenecientes a este período, guardan, curiosamente, alguna relación con este tipo de lugares, salvo en algunos casos de hallazgos aislados sin contexto cuyo origen es oscuro, al proceder de colecciones privadas. No obstante, en algunos de 
estos casos, hemos podido documentar igualmente su procedencia acuática, como ocurre, por ejemplo, con los objetos que tratamos.

Hasta ahora el número de hallazgos y depósitos localizados en zonas acuáticas de la Península Ibérica pertenecientes al Bronce Final asciende a unos 32 aproximadamente, de los cuales 12 son andaluces. A pesar de que el número de localizaciones es menor en Andalucía debido a que, en gran parte, estos hallazgos no han sido declarados o se han perdido los datos referentes a su origen, el número global de objetos es mayor, contando además con el depósito más importante de los hallados hasta ahora en la Península Ibérica, como es el producido en la Ría de Huelva en el primer tercio del presente siglo.

En los depósitos acuáticos andaluces, los objetos más importantes son las espadas, que aparecen en 12 de ellos: Ría de Huelva (Almagro Basch, 1975a), Alcalá del Río (Ruiz-Gálvez, 1984), La Cartuja, Bellavista, en Sevilla (Ruiz-Gálvez, 1984: 671), Vado de Mengíbar, Jaén (Ruiz-Gálvez, 1984: 671), Río Guadalete, Cádiz (Ruiz-Gálvez, 1984: 672), Coto de Doñana, Huelva (Caro Bellido; Lavado en prensa), Corta de la Cartuja, Sevilla (Ruiz-Gálvez, 1984: 671), Bornos, Cádiz (Ruiz-Gálvez, 1984: 672), Marmolejo, Jaén (Carrasco et alii, 1987: 103), Baeza, Granada (Carrasco et alii, 1987: 103), El Remanso de las Golondrinas, Sevilla (López Palomo, 1978: 233 y ss.) y en este mismo que estudiamos. Entre estos, las espada de lengüeta calada, aletas con horadaciones y hoja de lengua de carpa, aparece en todos los casos citados, excepto un ejemplar de Alcalá del Río y los de Mengíbar, La Cartuja y el Guadalete.

En los depósitos andaluces, al contrario de lo que suele ocurrir en el caso de los producidos en gran parte de la fachada atlántica, no aparecen hachas (Ruiz-Gálvez, 1984: 669 y ss.), pero sí otras armas, como puntas de lanza, regatones y otros objetos como fíbulas, etc.

En lo referente a la posible cronología de este depósito tenemos como únicos elementos válidos las espadas. Hasta ahora la falta de contextos arqueológicos en los hallazgos realizados, ha impulsado a los investigadores a recurrir a moldes tipológicos preestablecidos, basados en paralelos con otros ejemplares europeos o mediterráneos, como ha venido ocurriendo a partir del ya clásico estudio de M. Almagro Basch sobre las piezas de la Ría de Huelva (Almagro Basch, 1975b). Nuevos hallazgos en contexto, como el del Cerro de la Miel (Carrasco et alii, 1987) permitirán afinar y controlar más en el futuro los aspectos culturales y cronológicos de estos objetos.

Nuestra pieza difiere en algunos puntos del ejemplar granadino como, por ejemplo, en el número y forma de las horadaciones de la lengüeta y aletas, la distinta disposición de los gavilanes, la disposición del nervio central de la hoja, etc. Pero creemos que estas diferencias no son especialmente relevantes para una diferenciación cronológica clara, ya que son enormemente mayores los caracteres que las asemejan, que aquellos que las separan.

En la situación actual de la investigación sobre este tema, creemos que podemos llevar la cronología de esta espada y con ella la del depósito que comentamos, hasta mediados del siglo IX, sin que ello obvie la posibilidad de su existencia en momentos anteriores (Carrasco et alii, 1987). 


\section{BIBLIOGRAFIA}

Almagro Basch, M. (1939): «El hallazgo de la Ría de Huelva y el final de la Edad del Bronce en el Occidente de Europan. Ampurias II: 85-143.

Almagro GorbeA, M. (1972): "La espada de Guadalajara y sus paralelos peninsulares». Trabajos de Prehistoria 29: 55-78.

Burguess, C.; CoOmBs, D. (1972): «Bronze Age Hoards». Some finsd old and news. IV-V.

CARo BeluDo, A. (prensa): «Nueva espada de lengua de carpa en la costa de Huelvan. Gades.

Carrasco, A.; Pachón, M.; Gamiz. J. (1978): La espada del «Cerro de la Mora y su contexto arqueológico. Nuevas aportaciones para el conocimiento de la metalurgia del Bronce Final en el Sudeste Peninsular. Excmo. Ayuntamiento de Moraleda Zafayona. (Granada).

Coffyn, A. (1985): «Le Bronze Final Atlantique dans la Peninsule Iberiquen. Pub. Centre P. Paris. París. 431 pp.

Fernández Miranda, M.; Ruiz Gálvez, M. L. (1980): «El depósito de la Ría de Huelva y su contexto cultural». Oskitania, I. p. 66 ss.

López Palomo, J. A. (1978): „Pequeño depósito de bronces del Río Genil». Cuadernos de Prehistoria de la Universidad de Granada, 3: 233-240.

Ruiz Gálvez, M. L. (1984): La Península Ibérica y sus relaciones con el círculo cultural atlántico. Ed. Univ. Complutense. Madrid. 